Z Rheumatol 2021 $\cdot 80: 641-646$ https://doi.org/10.1007/s00393-021-01034-y Angenommen: 25. Mai 2021

Online publiziert: 1. Juli 2021

(c) Der/die Autor(en) 2021

\section{Redaktion}

U. Müller-Ladner, Bad Nauheim

U. Lange, Bad Nauheim

Rebecca Hasseli ${ }^{1}$ (D) - Alexander Pfeil ${ }^{2}$ Bimba Franziska Hoyer ${ }^{3} \cdot$ HannsMartin Lorenz ${ }^{4}$ - Anne C. Regierer ${ }^{5}$. Jutta G. Richter ${ }^{6} \cdot$ Tim Schmeiser $^{7}$. Anja Strangfeld ${ }^{5} \cdot$ Reinhard E. Voll $^{8} \cdot$ Andreas Krause $^{9} \cdot$ Hendrik Schulze-Koops $^{10}$. Ulf Müller-Ladner ${ }^{1}$. Christof Specker ${ }^{11}$

'Abteilung für Rheumatologie, Klinische Immunologie, Osteologie und Physikalische Medizin, JustusLiebig-Universität Gießen, Campus Kerckhoff, Bad Nauheim, Deutschland; ${ }^{2}$ Funktionsbereich Rheumatologie und Osteologie, Klinik für Innere Medizin III, Universitätsklinikum Jena, Jena, Deutschland; ${ }^{3}$ Sektion für Rheumatologie, 1. Medizinische Klinik, Universitätskrankenhaus Schleswig-Holstein Campus Kiel, Kiel, Deutschland; ' ${ }^{4}$ Sektion Rheumatologie, Medizinische Klinik V, Universitätsklinikum Heidelberg, Heidelberg, Deutschland; ${ }^{5}$ Programmbereich Epidemiologie und Versorgungsforschung, Deutsches Rheuma-Forschungszentrum, Berlin, Deutschland; ${ }^{6}$ Poliklinik, Funktionsbereich und Hiller Forschungszentrum für Rheumatologie, Universitätsklinikum Düsseldorf, Heinrich-Heine-Universität Düsseldorf, Düsseldorf, Deutschland; ${ }^{7}$ Rheumatologie im Veedel, Köln, Deutschland; ${ }^{8}$ Klinik für Rheumatologie und Klinische Immunologie, Universitätsklinikum Freiburg, Medizinische Fakultät, Universität Freiburg, Freiburg, Deutschland; ${ }^{9}$ Abteilung für Rheumatologie, Osteologie und Klinische Immunologie, Immanuel Krankenhaus Berlin, Berlin, Deutschland; ${ }^{10}$ Sektion Rheumatologie und Klinische Immunologie, Medizinische Klinik und Poliklinik IV, Ludwig-Maximilians-Universität München, München, Deutschland; " Klinik für Rheumatologie \& Klinische Immunologie, Kliniken Essen-Mitte, Essen, Deutschland

\title{
Deutsches Register www.Covid19-Rheuma.de
}

\section{Statusbericht nach 1 Jahr der Pandemie}

Mit dem Ausbruch des Severe Acute Respiratory Syndrome Coronavirus 2 (SARS-CoV-2 bzw. COVID-19) in Wuhan im Dezember 2019 entwickelte sich aus einem lokalen Infektionsgeschehen binnen 3 Monaten eine Pandemie [1]. Die Deutsche Gesellschaft für Rheumatologie (DGRh) veröffentlichte am 30.03.2020 erste, konsentierte Handlungsempfehlungen für die Betreuung ${ }^{1}$ mit entzündlich rheumatischen Erkrankungen (ERE) im Rahmen der COVID19-Pandemie, die sich naturgemäß noch nicht auf Studien $\mathrm{zu}$ Infektionen mit SARS-CoV-2 stützen konnten [2]. Basis hierfür waren Daten von bekannten viralen Infektionen der oberen und unteren Atemwege, z.B. mit Influenzaviren oder bekannten Coronaviren, wie SARS (schweres akutes respiratorisches Syndrom) oder MERS (Middle East

\footnotetext{
1 In dieser Arbeit wird aus Gründen der besseren Lesbarkeit das generische Maskulinum verwendet. Weibliche und anderweitige Geschlechteridentitäten werden dabei ausdrücklich mitgemeint, soweit es für die Aussage erforderlich ist.
}

Respiratory Syndrome), welche mit einer COVID-19 vergleichbaren initialen Klinik (Husten, Fieber, Zephalgien und Myalgien), demselben Übertragungsweg (Tröpfcheninfektion) und variabler Symptomatik, von symptomlos bis $\mathrm{zu}$ kurzen letalen Verläufen, einhergehen. Im Hinblick auf die immunmodulierende Therapie wurde von einem Pausieren oder einer Reduktion aus Sorge vor einer Infektion ausdrücklich abgeraten, da man - wiederum in Analogie zu bekannten Infektionen bei ERE - davon ausging, dass dadurch Krankheitsschübe begünstigt werden, die auch das Risiko für eine SARS-CoV-2-Infektion erhöhen dürften, spätestens wenn man zur Behandlung eines Schubes wieder höhere Glukokortikoiddosen (GC) einsetzen müsste [2]. Die Fortsetzung der immunmodulierenden Therapie setzt neben einer adäquaten und engmaschigen rheumatologischen Betreuung mit enger Arzt-Patienten-Kommunikation, auch eine entsprechende Compliance der Patienten voraus. In einer longitudinalen Befragung von Patienten aus rheumatologischen Ambulanzen und Praxen in
Deutschland über einen Zeitraum von 3 Monaten konnte gezeigt werden, dass die Mehrheit der Patienten angab, ihre Therapie gemäß der DGRh-Empfehlung fortzuführen [3].

Um Erkenntnisse zum adäquaten Umgang mit der immunmodulatorischen Therapie bei Patienten mit ERE im Kontext der COVID-19-Pandemie zu gewinnen, sind Registerdaten mit einer hohen Fallzahl sehr hilfreich. Diese erlauben, das Risiko für einen schweren

\begin{tabular}{|ll}
\hline \multicolumn{2}{|l}{ Abkürzungen } \\
\hline COVID-19 & Corona Virus Disease 2019 \\
\hline DGRh & $\begin{array}{l}\text { Deutsche Gesellschaft für } \\
\text { Rheumatologie }\end{array}$ \\
\hline GC & Glukokortikoide \\
\hline LEOSS & $\begin{array}{l}\text { Lean European Open Survey on } \\
\text { SARS-CoV-2 infected patients }\end{array}$ \\
\hline RA & Rheumatoide Arthritis \\
\hline $\begin{array}{l}\text { SARS- } \\
\text { CoV-2 }\end{array}$ & $\begin{array}{l}\text { Severe Acute Respiratory Syn- } \\
\text { drome Coronavirus 2 }\end{array}$ \\
\hline SpA & Spondyloarthritis \\
\hline
\end{tabular}


Infobox 1 Zugang zum Register und zur Impferfassung für Patienten

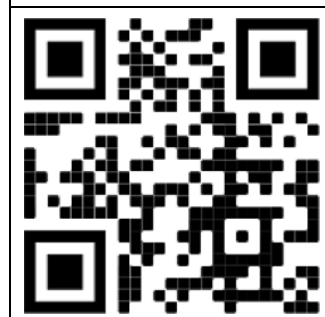

Zugang zum Register:

www.covid19-rheuma.de

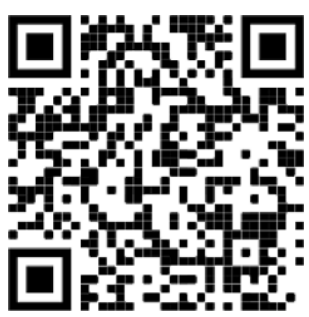

Zugang zur Impferfassung für Patienten: https://www.covid19-rheuma.de/patienteninformation-impfung

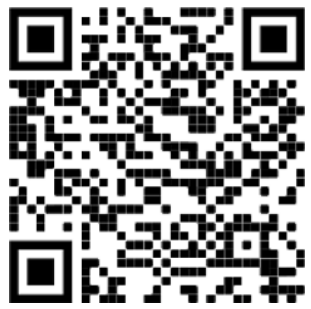

Zugang zur Papierversion der Impferfassung: https://www.covid19-rheuma.de/pdf/ fragebogen-impfung-20210413.pdf

Verlauf einer SARS-CoV-2-Infektion bei Patienten mit verschiedenen ERE oder unter einer bestimmten Therapie besser abzuschätzen. Aus diesem Grund initiierte die DGRh bereits im März 2020 gemeinsam mit der Justus-LiebigUniversität Gießen ein Online-Register (www.covid19-rheuma.de), mit dessen Hilfe nachgewiesene SARS-CoV-2-Infektionen (positiver PCR- oder Antikörpertest) bei Patienten mit ERE innerhalb weniger Minuten erfasst werden können. Im Register werden u. a. folgende Aspekte erfasst: Bundesland, Alter, Geschlecht, Gewicht, Größe, Impfstatus (Grippe-, Pneumokokken- und SARS-CoV-2Impfung), Komorbiditäten, Krankheitsaktivität und Therapie der rheumatischen Grunderkrankung zum Zeitpunkt der SARS-CoV-2-Infektion und deren Ver-

\begin{tabular}{lll}
\hline $\begin{array}{l}\text { Tab. } 1 \\
\text { Patientenverteilung nach den Bundesländern (Stand: 21.03.2021) }\end{array}$ & $\begin{array}{l}\text { DGRh COVID-19- } \\
\text { Register }\end{array}$ & $\begin{array}{l}\text { COVID-19-Fälle in der Allgemeinbevölke- } \\
\text { rung (laut Robert Koch-Institut) }\end{array}$ \\
\hline Bayern & $412(21 \%)$ & $472.427(18 \%)$ \\
Nordrhein-Westfalen & $262(13 \%)$ & $577.480(22 \%)$ \\
\hline Hessen & $241(12 \%)$ & $205.734(8 \%)$ \\
\hline Baden-Württemberg & $239(12 \%)$ & $343.165(13 \%)$ \\
\hline Berlin & $215(11 \%)$ & $138.205(5 \%)$ \\
\hline Sachsen & $148(7 \%)$ & $209.156(8 \%)$ \\
\hline Brandenburg & $116(6 \%)$ & $82.945(3 \%)$ \\
\hline Rheinland-Pfalz & $82(4 \%)$ & $109.639(4 \%)$ \\
\hline Hamburg & $71(4 \%)$ & $56.992(2 \%)$ \\
Niedersachsen & $69(3 \%)$ & $183.678(7 \%)$ \\
\hline Saarland & $66(3 \%)$ & $30.668(1 \%)$ \\
Schleswig-Holstein & $39(2 \%)$ & $46.969(2 \%)$ \\
Sachsen-Anhalt & $18(<1 \%)$ & $67.601(3 \%)$ \\
Thüringen & $18(<1 \%)$ & $87.286(3 \%)$ \\
\hline Mecklenburg-Vorpommern & $7(<1 \%)$ & $27.936(1 \%)$ \\
Bremen & $2(<1 \%)$ & $19.635(1 \%)$ \\
Gesamt & 2005 & 2.659 .516
\end{tabular}

lauf. Dieses bundesdeutsche Register soll auch dazu dienen, den Verlauf oder den Ausgang von COVID-19 bei Patienten mit ERE in Deutschland mit anderen Ländern vergleichen $\mathrm{zu}$ können, die sich z. B. im Hinblick auf die medizinische Versorgung und das jeweilige Gesundheitssystem unterscheiden [4].

\section{Vernetzung des COVID-19- Rheuma Registers}

Bereits bei der Konzeption des COVID19-Rheuma.de Registers wurde darauf geachtet, Inhalte und Datenbankstruktur so zu gestalten, dass die Daten auch mit anderen nationalen und internationalen Registern vergleichend ausgewertet werden können. Die Daten des deutschen Registers können so auch in das europäische Register (EULARCOVID-19-Registry) sowie in das internationale Register (COVID-19-Global Rheumatology Alliance) übertragen werden, sodass keine Doppeleingabe der deutschen Patienten in die internationalen Register nötig ist. Aus dieser Zusammenarbeit resultierte bereits eine weitere, wichtige Publikation zu Faktoren, die mit einer verstärkten Hospitalisierung bei COVID-19 und ERE assoziiert sind [5]. Neben der Verbindung zu den anderen rheumatologischen COVID-19-
Registern wurde eine Kooperation zwischen dem DGRh-Register und dem Lean European Open Survey on SARSCoV-2 infected patients(LEOSS)-Register aufgebaut, welches SARS-CoV-2Infektionen insgesamt europaweit erfasst [6]. Auf dieser Kooperation basierend, ist aktuell eine Analyse des Verlaufes einer SARS-CoV-2-Infektion bei rheumatologischen Patienten im Vergleich $\mathrm{zu}$ Patienten ohne ERE oder anderen Autoimmun-/Tumorerkrankungen geplant.

\section{Analysen aus dem COVID-19- Rheuma Register im ersten Jahr der Pandemie}

Im Rahmen der ersten Publikation zum COVID-19-Register erfolgten die Beschreibung des Aufbaus des DGRhRegisters sowie eine erste Analyse der Akzeptanz und Rekrutierung nach einem Dokumentationszeitraum von 4 Wochen [7]. In diesem Zeitraum, welcher v. a. die erste Welle der COVID-19-Pandemie in Deutschland umfasste, wurden $104 \mathrm{~Pa}$ tienten erfolgreich dokumentiert, und der Einsatz von GC kristallisierte sich als ein erster möglicher Risikofaktor für eine Hospitalisierung im Rahmen von COVID-19 heraus [7]. 
In der Folgepublikation mit zum damaligen Zeitpunkt 468 erfassten Patienten konnten Risikofaktoren hinsichtlich einer Hospitalisierung bei einer SARSCoV-2-Infektion sehr viel genauer analysiert werden [8]. Hierbei wurden Patientenalter, kardiovaskuläre Komorbiditäten, chronisch interstitielle Lungenerkrankungen bzw. chronisch obstruktive Lungenerkrankungen und der Einsatz von GC als unabhängige Faktoren für die Notwendigkeit einer stationären Behandlung von COVID-19 bei Patienten mit ERE identifiziert. Besonders hervorzuheben war, dass auch die Krankheitsaktivität der ERE als ein unabhängiger Prädiktor für eine Hospitalisierung identifiziert werden konnte [8]. Dies zeigte sich auch in der Analyse der globalen Registerdaten, bei der eine erhöhte Krankheitsaktivität signifikant assoziiert war mit COVID-19-bedingter Mortalität [9]. Hierdurch ließ sich die Empfehlung der DGRh, bestätigen, dass eine adäquat immunmodulatorisch therapierte ERE ein geringeres Risiko für eine Hospitalisierung bzw. einen schweren Krankheitsverlauf bei einer SARS-CoV-2-Infektion haben dürfte $[8,9]$. Eine rasch zunehmende Zahl von Publikationen zu COVID19 generell und speziell bei Patienten mit ERE, hier insbesondere auch aus den Registern, war für die DGRh dann auch Anlass, die ersten Handlungsempfehlungen nach systematischer Literaturrecherche bereits Mitte $2020 \mathrm{zu}$ aktualisieren [10].

\section{Aktueller Stand des Registers}

Aktuell sind im Register 2005 Patienten erfasst (Stand: 21.03.2021). Mehrheitlich stammen die eingeschlossenen Patienten aus den Bundesländern Bayern, Baden-Württemberg, NordrheinWestfalen und Hessen. Diejenigen Bundesländer, die in der Allgemeinbevölkerung höhere Zahlen von SARS-CoV2-Infektionen aufweisen, sind im rheumatologischen Register ebenfalls stärker vertreten (•Tab. 1). Das mittlere Alter der 1348 Frauen, 655 Männer und 2 diversgeschlechtliche Personen beträgt 65 Jahre (Range 19 bis 96 Jahre). Die Haupterkrankungsbilder stellen die rheumatoide Arthritis (RA, 46\%), die Spondyloarthritiden (SpA, 27\%) ein-

Z Rheumatol 2021 · 80:641-646 https://doi.org/10.1007/s00393-021-01034-y

(c) Der/die Autor(en) 2021

R. Hasseli · A. Pfeil · B. F. Hoyer · H.-M. Lorenz - A. C. Regierer · J. G. Richter · T. Schmeiser · A. Strangfeld · R. E. Voll · A. Krause · H. Schulze-Koops · U. Müller-Ladner · C. Specker

\section{Deutsches Register www.Covid19-Rheuma.de. Statusbericht nach 1 Jahr der Pandemie}

\section{Zusammenfassung}

Durch das COVID-19-Register (www.covid19rheuma.de) der Deutschen Gesellschaft für Rheumatologie erfolgte erstmalig die Erfassung und systematische Evaluation einer viralen Infektion bei Patienten mit entzündlich rheumatischen Erkrankungen (ERE). Hierdurch war und ist eine schnelle Generierung von wissenschaftlichen Daten möglich, welche helfen, die Betreuung von Patienten mit ERE im Rahmen der Pandemie zu verbessern. Neben der Bestätigung allgemeiner Risikofaktoren - auch für Patienten mit ERE - wie Patientenalter und Komorbiditäten (z. B. kardiovaskuläre, chronische Lungenund Nierenerkrankungen) konnten die Einnahme von Glukokortikoiden und die Krankheitsaktivität der rheumatischen Erkrankung als krankheitsspezifische Risikofaktoren für die Notwendigkeit einer stationären Behandlung wegen COVID-19 identifiziert werden. Auswertungen der kontinuierlich wachsenden Kohorte von Patienten mit entzündlich rheumatischen Erkrankungen und einer COVID-19-Infektion erlauben, Handlungsempfehlungen für die Betreuung der Patienten auf eine bessere Evidenz zu stützen. Die Kooperation mit internationalen rheumatologischen Registern (z. B. europäisches COVID-19-Register für ERE) ermöglicht Analysen aggregierter Kohortendaten von Patienten mit entzündlich rheumatischen Erkrankungen und einer SARS-CoV-2-Infektion für internationale Vergleiche und statistisch noch besser abgesicherte Aussagen.

Schlüsselwörter

Entzündlich-rheumatische Erkrankungen - Immunmodulation - SARS-CoV-2 . Glukokortikoide $\cdot$ Risikofaktoren

\section{German registry www.Covid19-Rheuma.de. Status report after 1 year of the pandemic}

\section{Abstract}

The COVID-19 registry (www.covid19rheuma.de) of the German Society of Rheumatology was the first registry for the acquisition and systemic evaluation of viral infections in patients with inflammatory rheumatic diseases (IRD). This has enabled rapid generation of scientific data that will help to improve the care of patients with IRD in the context of the pandemic. In addition to confirming general risk factors, such as patient age and comorbidities (e.g. cardiovascular, chronic lung and kidney diseases), the use of glucocorticoids and the disease activity of the rheumatic disease could be identified as disease-specific independent risk factors for the need of hospitalization due to COVID-19. Evaluations of the continuously growing cohort of patients with IRD and COVID-19 enable recommendations for patient care to be based on better evidence. Cooperation with international rheumatology registries (e.g. European COVID-19 registry for IRD) enables analyses of aggregated cohorts of patients with IRD and COVID-19 for international comparisons and statistically even more reliable statements.

\section{Keywords}

Inflammatory rheumatic diseases - Immunomodulation - SARS-CoV-2 - Glucocorticoids . Risk factors schließlich der Psoriasisarthritis und die Kollagenosen (12\%) dar. Des Weiteren weisen $3 \%$ der eingeschlossenen Patienten eine ANCA(antineutrophile zytoplasmatische Antikörper)-assoziierte Vaskulitis auf (• Abb. 1). Mehrheitlich wurden die Patienten mit Methotrexat therapiert, gefolgt von GC und TNFInhibitoren (- Abb. 2). Positiv zu ver- merken ist, dass aktuell 1630 Patienten bereits genesen sind, allerdings wurden auch 78 letale Verläufe gemeldet, die mehrheitlich in der zweiten Welle der Pandemie erfasst wurden (• Abb. 3). 


\section{Hot Topics}

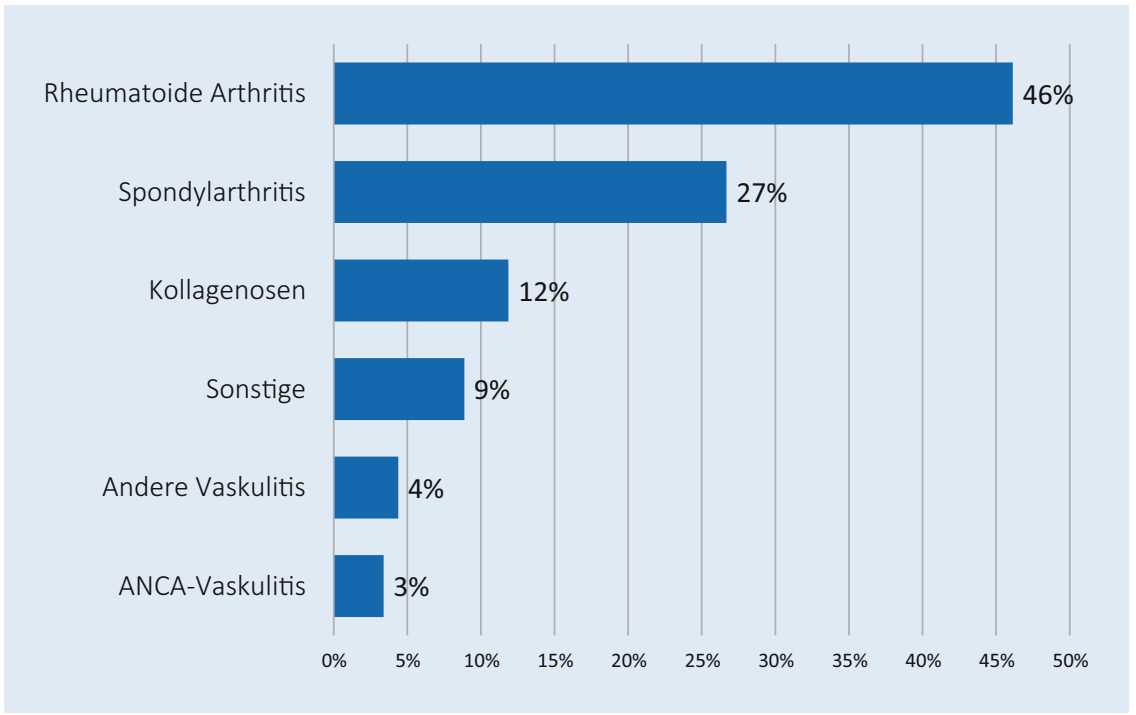

Abb. $1 \Delta$ RheumatischeErkrankungen ( $n=2005)$.Verteilung derhäufigsten Diagnosen/Diagnosegruppen in dem deutschen COVID19-Rheuma Register (Stand 21.03.2021). Doppelnennungen möglich bei Overlap-Syndromen

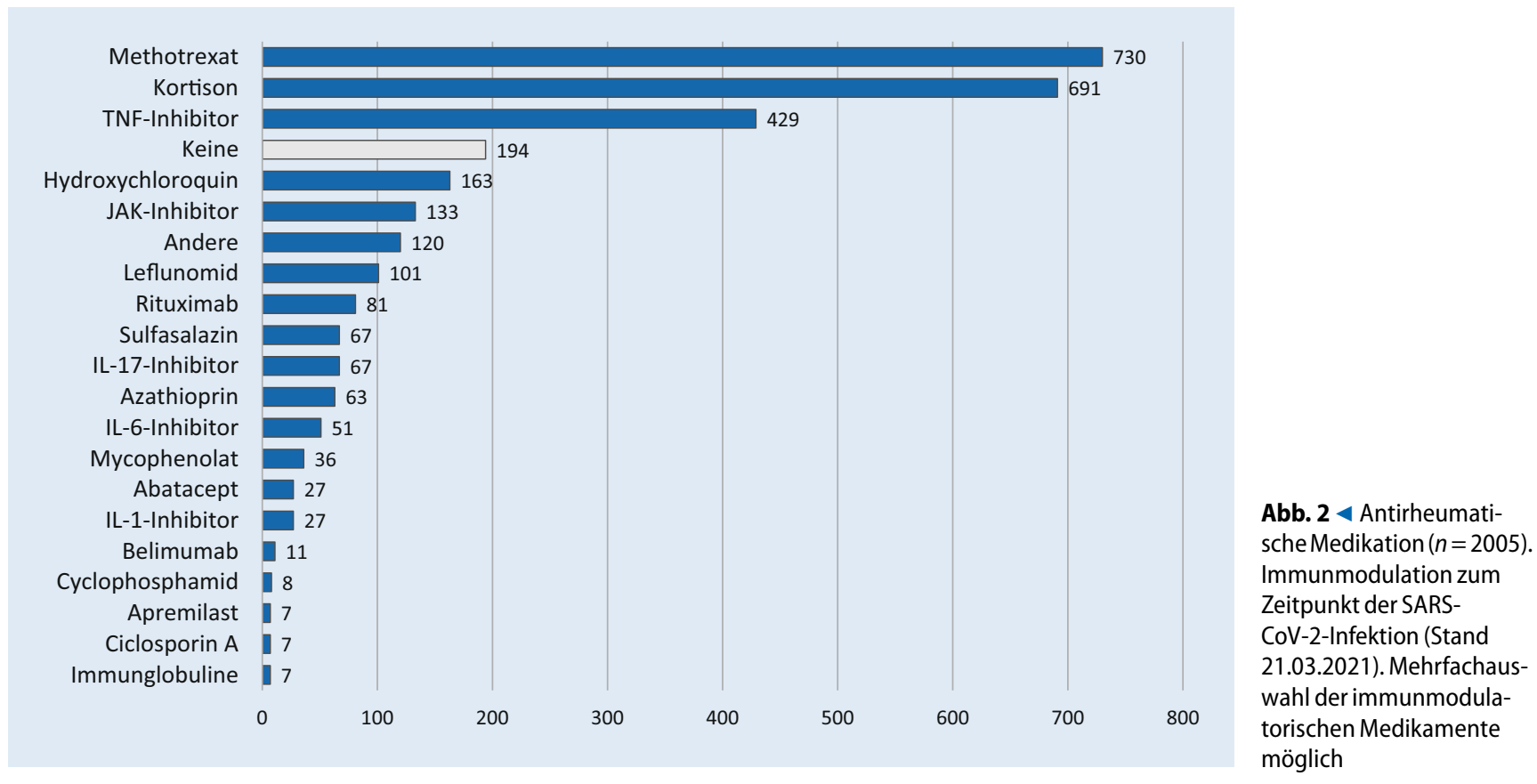

\section{Aktuelle Publikationen und weitere Projekte}

Zur Frage, inwieweit sich der Verlauf einer SARS-CoV-2-Infektion bei verschiedenen rheumatologischen Diagnosen unterscheidet, erfolgte in der aktuellsten Publikation aus dem Register eine erste Analyse der beiden größten Krankheitsgruppen: rheumatoide Arthritis (RA) und Spondyloarthritis (SpA) [11]. Hierbei wies die Gruppe der SpA eine niedrigere Hospitalisierungsrate (16\% vs. $30 \%$ ) auf. Dies könnte durch den geringeren Einsatz von GC bedingt sein, welche lediglich $13 \%$ der SpA-Patienten erhielten gegenüber $40 \%$ der RA-Patienten. Bezüglich der letalen Verläufe zeigte sich aber kein signifikanter Unterschied [11].

Flankierend wurde zu dem deutschen COVID-19-Register eine Online-Umfrage zu Auswirkungen der Corona-Pandemie auf Rheumapatientinnen und -pati- enten aufgesetzt, welche prospektiv über 1 Jahr versucht, die rheumatologische Versorgung und den Umgang der Patienten mit den Problemen, welche die Pandemie für diese mit sich bringt, $\mathrm{zu}$ erfassen. Zwischen April und Juli 2020 hatten sich insgesamt 695 Patienten eingeschrieben, sodass dieses Projekt im August 2021 ausgewertet werden kann.

Des Weiteren erfolgte im Februar und März 2021 eine Umfrage unter deutschen Rheumatologen, um deren Einstellung 


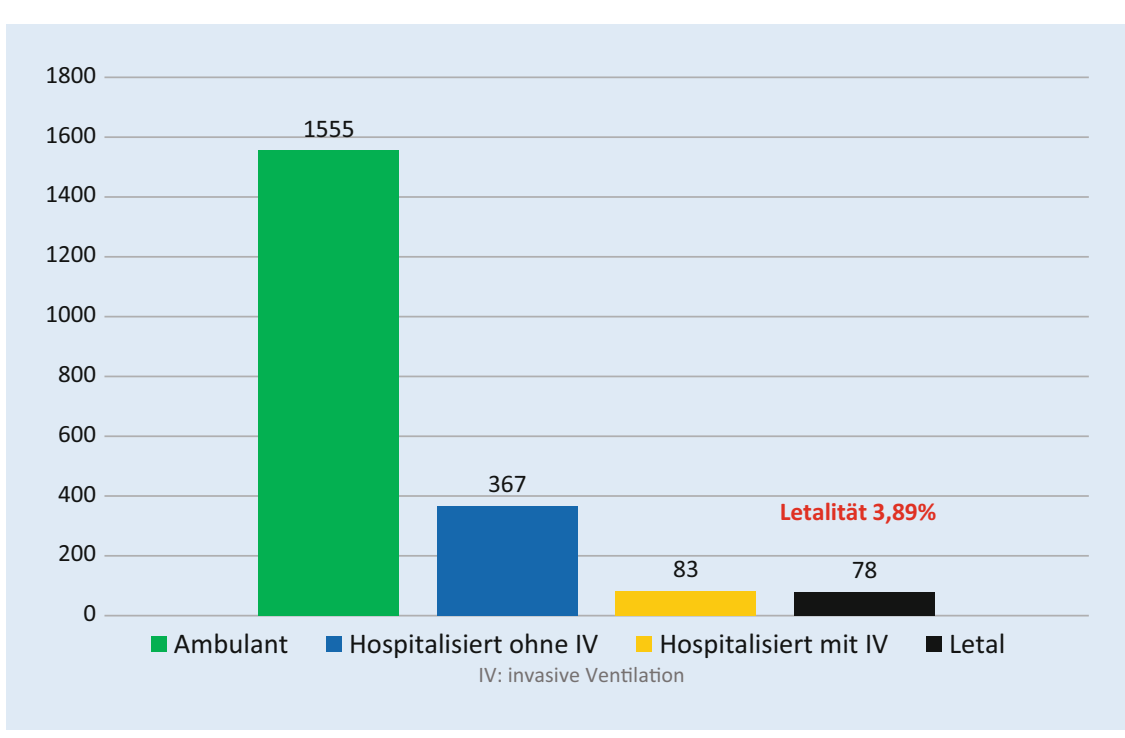

Abb. $3 \Delta$ Verläufe der SARS-CoV-2-Infektion im Rheumaregister. Letale Verläufe sind in hospitalisierte bzw. ambulante Patienten eingeschlossen

zur Corona-Impfung und zu dem Umgang mit Fragen ihrer Patienten zur Impfproblematik zu analysieren. Die Antworten von insgesamt 214 Ärztinnen und Ärzten werden derzeit ausgewertet.

Im Rahmen der aktuellen Aktivität der Ad-hoc-Kommission COVID-19 der DGRh erfolgt eine Erfassung von Corona-Impfungen bei Patienten mit ERE in einem separaten Impfregister. Darin werden Patienten mit ERE gebeten, Fragen zu Verträglichkeit, Sicherheit und Effektivität der COVID-19-Vakzinierung über einen Zeitraum von 12 Monaten in mehrfachen kurzen Online-Befragungen zu beantworten. Dieses Impfregister wurde Anfang Februar 2021 gestartet und umfasst bislang bereits 106 Patienten (Stand: 21.03.2021). Für Patienten, die nicht selbst an der Online-Impferfassung teilnehmen können, besteht die Möglichkeit einer papierbasierten Erhebung (Download des Formulars unter www.covid19-rheuma.de, D Infobox 1).

\section{Korrespondenzadresse}

\section{Dr. med. Rebecca Hasseli}

Abteilung für Rheumatologie, Klinische Immunologie, Osteologie und Physikalische Medizin, Justus-Liebig-Universität Gießen, Campus Kerckhoff

Benekestr. 2-8, 61231 Bad Nauheim, Deutschland

r.hasseli@kerckhoff-klinik.de
Danksagung. Die Erfassung der rheumatologischen Patienten mit einer SARS-CoV-2-Infektion im COVID19-Register ist und war nur durch die Arbeit der dokumentierenden Ärztinnen und Ärzte, der medizinischen Fachangestellten und Studienpersonal möglich. Aktuell dokumentieren 324 Kolleginnen und Kollegen, medizinische Fachangestellte und Studienpersonal aktiv in das COVID19-Register. An dieser Stelle ist es der Ad-hoc-Kommission COVID19 Register der DGRh ein ganz besonderes Anliegen, einen großen Dank an die Kolleginnen und Kollegen, medizinische Fachangestellten und Studienpersonal für die nicht vergütete Dokumentation der Patienten in das Register auszusprechen. Diese beispielhafte Aktivität der DGRh, durch die bereits wichtige Erkenntnisse aus der Pandemie für unsere Patienten gewonnen werden konnten, wäre ohne das große Interesse und Engagement unserer Kolleginnen und Kollegen nicht möglich gewesen. Besonderer Dank gilt: Dr. Fredrik Albach, Dr. Annette Alberding, Dr. Tobias Alexander, Prof. Dr. Rieke Alten, Dr. Susanne Amann, Dr. Christopher Amberger, Dr. Michaela Amberger, Dr. Bianka Andermann, Nils Anders, loana Ios, Dr. Peer Aries, Prof. Dr. Martin Aringer, Dr. Uta Arndt, Sarah Avemarg, Prof. Dr. Marina Backhaus, Prof. Dr. Christoph Baerwald, Dr. Erich Bärlin, Dr. Nora Bartholomä., Dr. Hans Bastian, Dr. Michael Bäuerle, Dr. Jutta Bauhammer, Dr. Christine Baumann, Prof. Dr. Heidemarie Becker, Dr. Klaus Becker, Dr. Michaela Bellm, Dr. Sylvia Berger, Dr. Gerhard Birkner, Prof. Dr. Norbert Blank, Daniel Blendea, Dr. Hans Bloching, Dr. Stephanie Böddeker, Dr. Susanne Bogner, Dr. Martin Bohl-Bühler, Sebastian Böltz, Dr. llka Bösenberg, Nicole Böttcher, PD Dr. Dr. Jan Brandt-Jürgens, Dr. Matthias Braun, Dr. Matthias Braunisch, Dr. Jan Phillip Bremer, Dr. Matthias Broll, Dr. Andreas Bruckner, Dr. Veronika Brumberger, Dr. Martin Brzank, Dr. Sahra Büllesfeld, Sandra Burger, Dr. Gamal Chehab, Dr. Michaela Christenn, Dr. Anne Claußnitzer, Prof. Dr. Kirsten de Groot, Dr. Elvira Decker, Dr. Frank Demtröder, Dr. Jacqueline Detert, Dr. Rainer Dörfler, Dr. Ines Dornacher, Dr. Elke Drexler, Dr. Edmund Edelmann, Dr. Roman Eder, Dr. Christina Eisterhues, Dr. Andreas Engel, Dr. Joachim Michael Engel, Dr. BriAndreica, Dr. Jan Andresen, Dr. Nikolaos Andriopou- gitte Erbslöh-Möller, Dr. Miriam Feine, PD Dr. Martin Feuchtenberger, Prof. Dr. Dr. Christoph Fiehn, PD Dr. Rebecca Fischer-Betz, Prof. Dr. Martin Fleck, Dr. Stefanie Freudenberg, Dr. Christian Fräbel, Dr. Petra Fuchs, Dr. Regina Gaissmaier, Dr. Ino Gao, Oliver Gardt, Dr. Georg Gauler, Dr. Katrin Geißler, Dr. Joachim Georgi, Dr. Jasmin Gilly, Yannik Gkanatsas, Dr. Cornelia Glaser, Agnes Gniezinski-Schwister, Dr. Rahel Gold, Dr. Norman Görl, Dr. Karl-Heinz Göttl, Dr. Beate Göttle, Dr. Anett Gräßler, Dr. Ricardo Grieshaber-Bouyer, Dr. Gisela Grothues, Dr. Mathias Grünke, Dr. Elizabeth Guilhon de Araujo, Dr. Florian Günther, Mirjam Haag, Dr. Linda Haas, Dr. Anna Haas-Wöhrle, Dr. Denitsa Hadjiski, PD Dr. Hildrun Haibel, Till Ole HallmannBöhm, Dr. Urs Hartmann, Dr. Charlotte S. Hasenkamp, Dr. Maura-Maria Hauf, Dr. Matthias Hauser, Dr. Nicole Heel, Dr. Liane Hein, Dr. Reinhard Hein, Dr. Claudia Hendrix, Prof. Dr. Jörg Henes, Dr. Karen Herlyn, Dr. Walter Hermann, Prof. Dr. Peter Herzer, Dr. Andrea Himsel, Dr. Guido Hoese, PD Dr. Paula Hoff, Marie-Therese Holzer, Dr. Johannes Hornig, Melanie Huber, Dr. Georg Hübner, Dr. Georg Hübner, Ole Hudowenz, PD Dr. Axel Hueber, Dr. Verena Hupertz, Dr. Elke Iburg, Dr. Annette Igney-Oertel, Dr. Steffen Illies, PD Dr. Annett M. Jacobi, Ilona Jandova, Dipl.Med. Christiane Jänicke, Dr. Sebastian T. Jendrek, Dr. Anne Johannes, Dr. Aaron Juche, Dr. Sarah Kahl, Dr. Ludwig Kalthoff, Dr. Wiebke Kaluza-Schilling, Dr. Eleni Kampylafka, Dr. Antje Kangowski, Dr. Andreas Kapelle, Dr. Kirsten Karberg, Dr. Dorothee Kaudewitz, Dr. Bernd Oliver Kaufmann, Prof. Dr. Gernot Keyßer, Dr. Nayereh Khoshraftar-Yazdi, Dr. Matthias Kirchgässner, Dr. Matthias Kirrstetter, Dr. Birgit Kittel, Dr. Christoph Kittel, Julia Kittler, Dr. Arnd Kleyer, Dr. Claudia Klink, Dr. Barbara Knau, Prof. Dr. Christian Kneitz, Anna Knothe, Dr. Katrin Köchel, Dr. Benjamin Köhler, Dr. Peter Korsten, Dr. Magdolna Kovacs, Dr. Dietmar Krause, Dipl.-Med. Gabi Kreher, Dr. Rene Kreutzberger, Dr. Eveline Krieger-Dippel, Prof. Dr. Klaus Krüger, Dr. Brigitte Krummel-Lorenz, Dr. Martin Krusche, Dr. Holger Kudela, Dr. Christoph Kuhn, Dr. Kerstin Kujath, Dr. Reiner Kurthen, Dr. Rolf Kurzeja, Peter Kvacskay, Prof. Dr. Peter Lamprecht, Sabine Langen, Dr. Heiko Lantzsch, Dr. Petra Lehmann, Dr. Nicolai Leuchten, PD Dr. Christian Löffler, Dr. Dorothea Longerich-Scheuß, Dr. Gitta Lüdemann, Dr. Thomas Lutz, Vanessa Maerz, Dr. Hartmut Mahrhofer, Dr. Ingeborg Maier, Prof. Dr. Karin Manger, Prof. Dr. Elisabeth Märker-Hermann, Dr. Anette Märtz, Dr. Anette Märtz, Hanin Matar, Dr. Johannes Mattar, Dr. Sebastian Maus, Dr. Ursula MaußEtzler, Dr. Regina Max, Dr. Florian Meier, Dr. Adelheid Melzer, Carlos Meneses, Dr. Hans-Jürgen Menne, Dr. Helga Merwald-Fraenk, Dr. Claudia Metzler, Dr. Sabine Mewes, Dr. Harriet Morf, Dr. Harald Mörtlbauer, Dr. Markus Mortsch, Dr. Burkhard Muche, PD Dr. Niels Murawski, Dr. Antoine Murray, Dr. Jana Naumann, Dr. Anabell Nerenheim, Dr. Joachim Neuwirth, Phuong Nguyen, Dr. Stine Niehus, Dr. Martin Nielsen, Dr. Matthias Noehte, Dr. Dirk Nottarp, Dr. Dieter Nüvemann, Dr. Wolfgang Ochs, Dr. Sarah Ohrndorf, Dr. Jürgen Olk, Dr. Silke Osiek, Dr. Filiz Özden, Dr. Bettina Panzer, Dr. Alina Patroi, Dr. Ulrich Pfeiffer, Dr. Dorothea Pick, Dr. Marta Piechalska, Dr. Matthias Pierer, I. Pohlenz, Dr. Mikhail Protopopov, Dr. Almut Pulla, Dr. Michael Purschke, Dr. Judith Rademacher, Dr. Wolf Raub, PD Dr. Jürgen Rech, Dr. Sabine Reckert, Dr. Sabine Reckert, Dr. Anke Reichelt de Tenorio, Dr. Christiane Reindl, Dr. Annja Reisch, Prof. Dr. Gabriela Riemekasten, PD Dr. Dr. Markus Rihl, Dr. Viale Rissom, Dr. Karin Rockwitz, Dr. Maike Rösel, Dr. Markus Röser, Dr. Christoph Rossmanith, PD Dr. Ekkehard Röther, Dr. Fabian Röther, Dr. Maria Roth-Szadorski, Prof. Dr. Martin Rudwaleit, Dr. Petra Saar, Jasemine Saech, Dr. Oliver Sander, Dr. Eva Sandrock, Dr. Ertan Saracbasi- 
Zender, Dr. Michael Sarholz, Dr. Christoph Schäfer, Dr. Kerstin Schäfer, Dr. Martin Scheel, Dr. Stefan Schewe, Dr. Hermine Schibinger, Magnus Schiebel, Dr. Andreas Schieweck-Güsmer, Dr. Susanne Schinke, Dr. Ulrike Schlenker, Dr. Daniel Schlittenhardt, PD Dr. Marc Schmalzing, Dr. Verena Schmitt, Dr. Matthias Schmitt-Haendle, Dr. Sebastian Schnarr, Dr. Dieter Schöffel, Dr. Michaela Scholz, Dr. Jutta Schönherr, Dr. Ulrich Schoo, Dr. Judith Schreiber, Anna-Sophie Schübler, Dr. Florian Schuch, Dr. llka Schwarze, Dr. Carola Schwerdt, Dr. Eva Seipelt, Dr. Matthias Sekura, Dr. Jörg Sensse, Dr. Nyamsuren Sentis, Dr. Christine Seyfert, Ondrej Sglunda, Dr. Naheed Sheikh, Dr. Iris Sievert, Dr. David Simon, Marta Sluszniak, Dr. Katharina Sokoll, Dr. Sigrid Sonn, Dr. Susanna SpäthlingMestekemper, Dr. Lydia Spengler, Gerald Stapfer, Dr. Nicolai Steinchen, Dr. Mirko Steinmüller, Karen Steveling, Dr. Karin Stockdreher, Dr. Helga Streibl, Prof. Dr. Johannes Strunk, Dr. Mechthild Surmann, Dr. Ingo H. Tarner, Dr. Stefanie Tatsis, Dr. Astrid Thiele, Dr. Jan Thoden, Dr. Anika Tuleweit, Prof. Dr. Peter Vaith, Dr. Inka Vallbracht-Ackermann, Dr. Susanne Veerhoff, Dr. Susanne Veerhoff, Prof. Dr. Nils Venhoff, Dr. Anita Viardot, Lisa Vinnemeier-Laubenthal, Dr. Markus Voglau, Dr. Marcus von Deimling, Dr. Cay-Benedict von der Decken, Dr. Heike von Löwis, Dr. Marisa Walther, Dr. Sven Weidner, Dr. Martin Weigelt, Prof. Dr. Stefan Weiner, Dr. Jutta Weinerth, Dr. Angela Weiß, Dr. Martin Welcker, Dr. Stephanie Werner, Dr. Dirk Wernicke, Dr. Franziska Wiesent, Prof. Dr. Peter Willeke, Dr. Lea Winau, Dr. Hans Wisseler, Dr. Matthias Witt, Dr. Stefan Wolf, Dr. Nina Wysocki, Dr. Panagiota Xanthouli, Dr. Monika Zaus, Dr. Markus Zeisbrich, Dr. Silke Zinke

Förderung. R. Hasseli wurde bei der Umsetzung des Registers durch das Clinician Scientist-Programm der Justus-Liebig Universität Gießen (JLU-CAREER) gefördert.

Funding. Open Access funding enabled and organized by Projekt DEAL.

\section{Einhaltung ethischer Richtlinien}

Interessenkonflikt. R. Hasseli, A. Pfeil, B.F. Hoyer, H.-M. Lorenz, A.C. Regierer, J.G. Richter, T. Schmeiser, A. Strangfeld, R.E. Voll, A. Krause, H. Schulze-Koops, U. Müller-Ladner und C. Specker geben an, dass in Bezug auf die Arbeit am COVID19-Rheuma.de Register und dieser Publikation kein Interessenkonflikt besteht.

Für die Durchführung der Registerarbeit liegt ein positives Ethikvotum der Justus-Liebig-Universität Gießen (\#52-50) vor, und sie wurde im Register für klinische Studien registriert (EuDRACT 2020-00195821). Die Arbeit erfolgt gemäß der Deklaration von Helsinki.

Open Access. Dieser Artikel wird unter der Creative Commons Namensnennung 4.0 International Lizenz veröffentlicht, welche die Nutzung, Vervielfältigung, Bearbeitung, Verbreitung und Wiedergabe in jeglichem Medium und Format erlaubt, sofern Sie den/die ursprünglichen Autor(en) und die Quelle ordnungsgemäß nennen, einen Link zur Creative Commons Lizenz beifügen und angeben, ob Änderungen vorgenommen wurden.

Die in diesem Artikel enthaltenen Bilder und sonstiges Drittmaterial unterliegen ebenfalls der genannten Creative Commons Lizenz, sofern sich aus der Abbildungslegende nichts anderes ergibt. Sofern das be- treffende Material nicht unter der genannten Creative Commons Lizenz steht und die betreffende Handlung nicht nach gesetzlichen Vorschriften erlaubt ist, ist für die oben aufgeführten Weiterverwendungen des $\mathrm{Ma}$ terials die Einwilligung des jeweiligen Rechteinhabers einzuholen.

Weitere Details zur Lizenz entnehmen Sie bitte der Lizenzinformation auf http://creativecommons.org/ licenses/by/4.0/deed.de.

\section{Literatur}

1. Salzberger B, Buder F, Lampl BT, Ehrenstein B, Hitzenbichler F, Holzmann T et al (2020) Epidemiologie von SARS-CoV-2/COVID 19: Aktueller Stand.Gastroenterologe. https://doi.org/10.1007/ s11377-020-00479-y

2. Schulze-Koops H, Specker C, Iking-KonertC, Holle J, Moosig F, Krueger K (2020) Preliminary recommendations of the German Society of Rheumatology (DGRh eV) for the management of patients with inflammatory rheumatic diseases during the SARS-CoV-2/Covid-19 pandemic. Ann Rheum Dis. https://doi.org/10.1136/annrheumdis-2020217628

3. Hasseli R, Müller-Ladner U, Keil F, Broll M, Dormann A, Fräbel C et al (2021) The influence of the SARS-CoV-2 lockdown on patients with inflammatory rheumatic diseases on their adherence to immunomodulatory medication-across sectional study over 3 months in Germany. Rheumatology (Oxford). https://doi.org/10.1093/ rheumatology/keab230

4. John Hopkins University \& Medicine (2021) Coronavirus resource center. https://coronavirus. jhu.edu/map.html.Zugegriffen: 21 Mär 2021

5. Gianfrancesco M, Hyrich KL, Al-Adely S, Carmona L, Danila MI, Gossec L et al (2020) Characteristics associated with hospitalisation for COVID-19 in people with rheumatic disease: data from the COVID-19 Global Rheumatology Alliance physician-reported registry. Ann Rheum Dis. https://doi. org/10.1136/annrheumdis-2020-217871

6. Jakob CEM, Borgmann S, Duygu F, Behrends U, Hower M, Merle U et al (2021) First results of the "Lean European Open Survey on SARS-CoV2-Infected Patients (LEOSS)". Infection 49:63-73. https://doi.org/10.1007/s15010-020-01499-0

7. Hasseli R, Mueller-Ladner U, Schmeiser T, Hoyer BF, Krause A, Lorenz H-M et al (2020) National registry for patients with inflammatory rheumatic diseases (IRD) infected with SARS-CoV-2 in Germany (ReCoVery): a valuable mean to gain rapid and reliable knowledge of the clinical course of SARS CoV-2 infections in patients with IRD. RMD Open 6:e1332. https://doi.org/10.1136/rmdopen-2020 001332

8. Hasseli R, Mueller-Ladner U, Hoyer BF, Krause A, Lorenz H-M, Pfeil A et al (2021) Older age, comorbidity, glucocorticoid use and disease activity are riskfactors for COVID-19hospitalisation in patients with inflammatory rheumatic and musculoskeletal diseases. RMD Open. https://doi. org/10.1136/rmdopen-2020-001464

9. Strangfeld A, Schäfer M, Gianfrancesco MA, Lawson-Tovey S, Liew JW, Ljung L et al (2021) Factors associated with COVID-19-related death in people with rheumatic diseases: results from the COVID-19 Global Rheumatology Alliance physician-reported registry. Ann Rheum Dis. https://doi.org/10.1136/ annrheumdis-2020-219498
10. Schulze-Koops $\mathrm{H}$, Iking-Konert $\mathrm{C}$, Leipe J, Hoyer BF Holle J, Moosig F et al (2020) Handlungsempfehlungen der Deutschen Gesellschaft für Rheumatologie e. V. für die Betreuung von Patienten mit entzündlich rheumatischen Erkrankungen im Rahmen der SARS-CoV-2/COVID-19-Pandemie - Update Juli 2020. Z Rheumatol. https://doi.org/ 10.1007/s00393-020-00851-x

11. Hasseli R, Pfeil A, Hoyer BF, Krause A, Lorenz HM, Richter JG et al (2021) Do patients with rheumatoid arthritis show a different course of COVID-19 compared to patients with spondyloarthritis? Clin ExpRheumatol 39(3):639-647 